\title{
Vecchie e nuove ipotesi sul primitivo mosaico della cupola della Cappella Palatina d'Aquisgrana
}

Simone Piazza

\section{(2) OpenEdition \\ Journals}

Edizione digitale

URL: http://journals.openedition.org/edl/1147

DOI: 10.4000/edl.1147

ISSN: 2296-5084

\section{Editore}

Université de Lausanne

\section{Edizione cartacea}

Data di pubblicazione: 15 mai 2018

Paginazione: 167-186

ISBN: 978-2-940331-68-0

ISSN: 0014-2026

Notizia bibliografica digitale

Simone Piazza, « Vecchie e nuove ipotesi sul primitivo mosaico della cupola della Cappella Palatina d'Aquisgrana ", Études de lettres [Online], 2 | 2018, online dal 15 mai 2020, consultato il 12 décembre 2020. URL : http://journals.openedition.org/edl/1147 ; DOI : https://doi.org/10.4000/edl.1147 


\section{VECCHIE E NUOVE IPOTESI \\ SUL PRIMITIVO MOSAICO DELLA CUPOLA DELLA CAPPELLA PALATINA D’AQUISGRANA}

Il contributo si interroga sull'assetto del primitivo mosaico della cupola della Cappella Palatina di Aquisgrana (inizi del IX secolo), contenente i ventiquattro Seniores dell'Apocalisse in adorazione di una Majestas Domini. Distrutta nel 1719, l'opera venne rifatta à l'identique negli anni 1879-1881, sulla base di esigui resti del disegno preparatorio, della lettura di Peter von Beeck (1620) e di quella di Giovanni Ciampini (1690), includente una preziosa incisione. L'incompletezza e la non piena convergenza di tali informazioni sono all'origine di alcune inesattezze riscontrabili nell'intervento di ripristino. Ulteriori imprecisioni della copia ottocentesca emergono da appunti e disegni di Palamède Fabri (1607-1608), pubblicati da Jean Hubert nel 1936, documenti che consentono, per altro, di riesaminare la controversa questione del perduto soggetto zenitale.

Se osservata dall'esterno, la Cappella Palatina di Aquisgrana, eretta intorno all'anno 800 per volere di Carlo Magno ${ }^{1}$, appare profondamente alterata da aggiunte e superfetazioni in stile gotico e barocco. Internamente, invece, l'edificio altomedievale ha più o meno mantenuto la sua configurazione primitiva, tanto nell'assetto architettonico dell'invaso ottagonale, culminante nella volta a padiglione, che nel disporsi della compagine decorativa, in marmo policromo e mosaici ${ }^{2}$. La natura

I. M. D'Onofrio, Roma e Aquisgrana, p. 101-144; L. Falkenstein, "Charlemagne et Aix-la-Chapelle»; G. Binding, "Die Aachener Pfalz Karls des Grossen als archäologisch-baugeschichtliches Problem»; S. Vanderputten, «La Chapelle Palatine à Aix-la-Chapelle et l'héritage classique».

2. Sui mosaici di Aquisgrana, portati a termine, verosimilmente, entro 1805, anno in cui l'edificio venne consacrato da papa Leone III, si veda: H. Schrade, 
di questi ultimi, tuttavia, non è affatto autentica, dal momento che i rivestimenti della cupola e del tamburo, così come quelli del deambulatorio e della tribuna, sono frutto di interventi di rifacimento ex novo. Il mosaico della volta è infatti opera della ditta veneziana di Antonio Salviati intervenuta fra il 1879 e il $1881^{3}$, mentre il resto della decorazione musiva è frutto di una squadra di artisti operanti negli anni 18881913 sotto la guida di Hermann Schaper ${ }^{4}$.

Quanto il nuovo mosaico della cupola si avvicini, iconograficamente, all'originale è problema arduo e dibattuto, visto che al tempo del ripristino tardo ottocentesco la decorazione carolingia mancava ormai da un secolo e mezzo. Il rivestimento primitivo, per la precisione, era andato distrutto nel 1719, a seguito della decisione di sostituirlo con ornamentazioni in stucco $^{5}$, vuoi per via del probabile deterioramento dell'antico tessuto musivo, vittima di un incendio nel $1656^{6}$, vuoi per un'esigenza di carattere estetico, legata alla scelta di introdurre una veste ornamentale più consona al gusto dell'epoca. Nei limiti di spazio consentiti in questa sede, il presente contributo si propone di riesaminare la questione dell'originario assetto figurativo della cupola di Aquisgrana, alla luce delle fonti e dei principali studi esistenti sull'argomento, focalizzando l'attenzione, in particolare, sul perduto tema zenitale?

Sappiamo che i modelli utilizzati per il nuovo mosaico della cupola furono messi a punto da un comitato internazionale di architetti,

"Zum Kuppelmosaik der Pfalzkapelle und zum Theoderich-Denkmal in Aachen»; R. Zanotto, "Mosaici, e sectilia, reimpiegati da Ravenna ad Aquisgrana»; U. Wehling, Die Mosaiken im Aachener Münster und ihre Vorstufen; A. von Euw, "Karl der Grosse als Schüler Alkuins, das Kuppelmosaik des Aachener Domes und das Maiestasbild in Codex C 80 der Zentralbibliothek Zürich", p. 8-15.

3. E. G. Grimme, Dom zu Aachen, p. 339; P. Clemen, Die romanische Monumentalmalerei in den Rheinlanden, p. 25; H. Belting, "Das Aachener Münster im 19. Jahrhundert»; U. Wehling, Die Mosaiken im Aachener Münster und ihre Vorstufen, spec. p. 79-81.

4. P. Heckes, «Die Mosaiken Hermann Schapers im Aachener Münster».

5. X. Barbier de Montault, "La mosaïque du Dôme à Aix-la-Chapelle», p. 289; P. Clemen, Die romanische Monumentalmalerei in den Rheinlanden, p. 26; J. Hubert, "Séance du 29 avril», p. 132.

6. X. Barbier de Montault, «La mosaïque du Dôme à Aix-la-Chapelle», p. 289.

7. La questione riguardante il tema zenitale dell'originaria decorazione musiva della Cappella Palatina di Aquisgrana rientra in un più ampio studio, di recente pubblicazione, dedicato all'area sommitale delle cupole e delle absidi d'età medievale: S. Piazza, Allo zenit della cupola. 
iconografi e archeologi, nominato dal governo prussiano nel 1869, cui fecero parte il tedesco Wilhelm Salzenberg (1803-1887), i francesi Alfred de Surigny (1805-1878) e Xavier Barbier de Montault (1830-1901), l'austriaco Friedrich von Schmidt (1825-1891), l'inglese John Henry Parker (1806-1884), l'italiano Pietro Ercole Visconti (1802-1880) e il belga Jean-Baptiste Bethune (1821-1894) ${ }^{8}$. A quest'ultimo, esponente di punta dello stile neomedievale, venne affidato il compito di presentare il programma figurativo per la realizzazione dell'opera musiva, prima in forma di bozzetti ad acquerello, poi, una volta approvato il progetto, mediante cartoni in scala 1 a $1^{\text {? }}$.

Relativamente alle volte anulari e al tamburo, la mancanza quasi totale di informazioni riguardanti i soggetti originari ${ }^{10}$ diede luogo alla scelta di temi di pura invenzione, ispirati soprattutto al repertorio figurativo dei mosaici ravennati ${ }^{11}$. Per la restituzione del programma iconografico della cupola, invece, fu possibile contare su alcuni frammenti

8. J.-B. Bethune D’Ydewalle, «Notes sur le projet de restauration de la mosaïque dans la coupole du dôme d'Aix-la-Chapelle», p. 40, n. 2; X. Barbier de Montault, «La mosaïque du Dôme à Aix-la-Chapelle», p. 287-289, 321-323.

9. "Mes honorables collègues du jury [...] voulurent bien m'engager avec instance, à en faire l'objet d'une étude approfondie et la base du projet de la restauration désirée. [...] Ayant pourtant pris la détermination de tenter la tâche difficile qu'on m'offrait avec tant de bienveillance, je fis à l'échelle de $1 / 24^{\text {me }}$ un dessin en aquarelle pour la recomposition de la mosaïque dans la voûte de la coupole. À ma grande surprise, ce projet reçut bientôt non seulement l'approbation du Chapitre d'Aix-la-Chapelle, mais encore celle du Karlsverein et des autorités ministérielles de Berlin. Je fus alors chargé de préparer les cartons en grandeur d'exécution»: J.-B. Bethune D’Ydewalle, "Notes sur le projet de restauration de la mosaïque dans la coupole du dôme d'Aix-la-Chapelle", p. 42. Sul ruolo avuto da Jean-Baptiste Bethune nella campagna di studio e rifacimento della decorazione musiva di Aquisgrana, cfr. U. Wehling, Die Mosaiken im Aachener Münster und ihre Vorstufen, p. 70-75.

Io. L'unica vaga notizia in proposito, riportata da Peter von Beeck al principio del XVII secolo (cfr. infra nel testo), riguarda l'ipotetica presenza di episodi dell'antico e del nuovo testamento: "Est et aliud quod fatigabit magis quam explebit intuentium acies, neque observatu indignum, templum nempe introrsum picturis in musivo seu musaico opere diversis typicis coloribus variegato repraesentante veteris ac novae legis acta quondam undequaque obvestitum incrustatumque fuisse, quod quidem obscuris ex concameratione vestibuli templi ad Lupinas valuas perspectius e nonnullarum fenestrarum absidibus perfectissime ex tholo ac triumphalis convexura fornicis turris primariae quae supra coronam pensilem in umbelico Basilicae aspectui se objicit»: P. von Beeck, Aquisgranum, p. 50.

II. P. Heckes, "Die Mosaiken Hermann Schapers im Aachener Münster», p. 191193 e 209 sq. 
Fig. 1 - G. G. Ciampini, Vetera Monimenta (1699), incisione raffigurante il mosaico della cupola della Cappella Palatina di Aquisgrana.

della sinopia e del disegno preparatorio dei mosaici carolingi, scoperti al momento della rimozione degli stucchi ${ }^{12}$, ma soprattutto sulle

I2. «Plusieurs membres du jury furent alors convoqués de nouveau: ils furent satisfaits de retrouver la construction carolingienne en bon état de conservation [...]. Dans la calotte de la coupole, les traits étant ça et là moins frustes qu'ailleurs, permirent de retrouver la silhouette de quelques figures et de constater leurs proportions: elles se rapportaient évidemment à la grande scène apocalyptique que Charlemagne avait fait représenter en mosaïque et dont la tradition populaire n'avait pas perdu le souvenir»: J.-B. Bethune D’Ydewalle, «Notes sur le projet de restauration de la mosaïque dans la coupole du dôme d'Aix-la-Chapelle», p. 42. Si vedano, a tale proposito, le note e le copie ad acquarello pubblicate da Paul Clemen (P. Clemen, Die romanische Monumentalmalerei in den Rheinlanden, p. 26 e n. 53, fig. 8 e tav. I), e l'analisi attenta e dettagliata di Ulrike Wehling, che fa riferimento al rinvenimento di frammenti pittorici di tipo diverso, ora tendenti al monocromo ora policromi, e prende perciò in considerazione la possibilità dell'esistenza, non solo di resti del disegno preliminare per la stesura del mosaico, ma anche di brani superstiti di un vero e proprio strato di intonaco dipinto, inteso come opera compiuta eseguita all'indomani della costruzione della cupola, prima che si decidesse di realizzare la campagna musiva, secondo un'ipotesi già avanzata da alcuni autori in precedenza (U. Wehling, Die Mosaiken im Aachener Münster und ihre Vorstufen, p. 23-27). A tale proposito riteniamo più probabile che 
abbondanti informazioni offerte da due fonti seicentesche: la testimonianza oculare di Peter von Beeck, canonico di Aquisgrana, autore di un'opera monografica riguardante la storia della sua città ${ }^{13}$, e le annotazioni di Giovanni Ciampini, contenute nei Vetera monimenta e corredate da una preziosa incisione ${ }^{14}$ (fig. 1). Dati e informazioni che consentirono ai mosaicisti veneziani di realizzare una copia più o meno fedele del programma originario della calotta.

Il tema è quello dei ventiquattro Seniores dell'Apocalisse (Ap. IV, 4 ; V), motivo iconografico in circolazione fin dal $\mathrm{V}$ secolo ${ }^{15}$, ampiamente diffuso in epoca carolingia, come attestato, per esempio, dai mosaici romani di Santa Prassede (817-824) ${ }^{16}$, dal Codex Aureus di Sant'Emmerano (860-880) ${ }^{17}$, e dalla Bibbia di San Paolo Fuori le

l'eterogeneità dei frammenti pittorici rinvenuti nel corso della rimozione della decorazione in stucco - oggi documentati soltanto dalle copie acquerellate ottocentesche dal momento che gli originali sono andati perduti - fosse dovuta a due diverse fasi esecutive entrambe appartenenti alla preparazione della stesura musiva: alludiamo cioè alla verosimile copresenza di resti di pennellate ocra dello schizzo disteso sull'arriccio ("sinopia»), e di brani del disegno realizzato sull'ultimo strato d'intonaco preliminarmente all'introduzione delle tessere ("disegno preparatorio»), eseguito con più colori $\mathrm{e}$ in modo più accurato del precedente abbozzo, secondo una prassi di antica e consolidata tradizione. Sulla tecnica del mosaico parietale nel Medio Evo e la distinzione fra sinopia e disegno preparatorio, cfr. P. J. Nordhagen, «Mosaico».

13. P. von Beeck, Aquisgranum, p. 50 sq.

I4. G. G. Ciampini, Vetera Monimenta, II, p. 134-135, tav. XLI.

I5. Si veda il mosaico romano dell'arco trionfale di San Paolo fuori le mura, rifatto dopo l'incendio del 1823 ma in maniera conforme all'originale del V secolo (G. Bordi, "Il busto di Cristo, i quattro simboli degli evangelisti, i ventiquattro Seniores e gli apostoli Pietro e Paolo", spec. p. 397 sq.), quello della facciata di San Pietro, ascrivibile agli anni di papa Leone Magno (440-461) e documentato da un disegno dell'XI secolo (G. Bordi, "L'Agnus Dei, i quattro simboli degli evangelisti e i ventiquattro Seniores nel mosaico della facciata di San Pietro in Vaticano») e infine quello dell'arco absidale dei Santi Cosma e Damiano, risalente, verosimilmente, al pontificato di Felice IV (526-530), conservatosi solo parzialmente: J. Osborne, «The Jerusalem temple treasure and the church of Santi Cosma e Damiano». Sul rapporto fra il tema dei Vegliardi dell'Apocalisse e l'immagine della Majestas Domini nell'arte paleocristiana e carolingia, cfr. A.-O. Poilpré, Maiestas Domini, p. 97-104.

I6. E. Thunø, " "Living stones" of Jerusalem».

I7. Monaco, Bayerische Staatsbibliothek, Clm 14000, f. 6r. Nel codice di Sant'Emmerano l'immagine dei Vegliardi - adoranti non il Cristo ma l'Agnello - è stata realizzata in pendant con l'illustrazione della pagina precedente, raffigurante Carlo il Calvo in trono all'interno di un loggiato (f. $5 \mathrm{v}$ ). Il legame fra le due miniature, sottolineato dal fatto che il monarca sembra contemplare l'Agnus Dei, è 
Fig. 2 - Aquisgrana, Cappella Palatina, veduta d'insieme della cupola.

\section{Mura (870 ca.) ${ }^{18}$. La versione odierna di Aquisgrana, ad imitazione} di quella primitiva, occupa l'intera superficie degli otto spicchi della

stato interpretato come un'allusione al tema apocalittico della cupola della Cappella Palatina di Aquisgrana dispiegantesi di fronte a Carlo Magno seduto nella tribuna ovest: J. Hubert, «Séance du 29 avril», p. 134; D. Alibert, «La majesté sacrée du roi»; A.-O. Poilpré, Maiestas Domini, p. 267. La miniatura dei Seniores contenuta nel Codex Aureus ha come titulus un carme di Alcuino alludente al medesimo tema apocalittico, associato, in questo caso, a Carlo Magno in persona: "Omnia quae praesens tellus producit alendo, / Et maris haec facies limbo circumvenit amplo, / Agne, Deum solio semper venerantur in alto. / Sanguine qui fuso tersisti crimina secli, / In cruce, tu Karoli detergas vulnera regis. / Cana caterva cluens vatum et venerabilis ordo, / Coetus apostolicus sertis caelestibus instans / Laudat, adorat, amat, devoto pectore temet / Et princeps Karolus vultu speculatur aperto, / Orans, ut tecum vivat longevus in aevum" (in M. G. H., Poetae Latini Aevi Carolini, III, Berlino, 1896, p. 254). Per un accostamento del carme di Alcuino al contesto del mosaico della cupola della Cappella Palatina, cfr. J. Hubert, «Séance du 29 avril», p. 134; P. E. Dutton, Charlemagne's mustache and other cultural clusters of a dark age, p. 94 sq.

I8. Roma, Basilica patriarcale di San Paolo f. 1. m., «Bibbia di San Paolo fuori le mura», f. 115v. Cfr. J. E. Gaehde, «La decorazione», p. 251 sq. 
volta a padiglione (fig. 2): su una fascia di prato paradisiaco sovrastata da un immenso cielo stellato, incedono, equidistanti, le solenni figure dei vegliardi, ritratte nell'atto di sollevare ciascuna la propria corona ${ }^{19}$ in direzione del Cristo della Seconda Venuta, collocato a metà altezza della cupola ${ }^{20}$, seduto su un trono ${ }^{21}$, a sua volta poggiante su un globo percorso da cerchi concentrici multicolori, a simboleggiare i cieli dell'universo; nella zona sommitale campeggiano i quattro simboli degli evangelisti disposti in volo attorno ad un motivo ombrelliforme.

Se nell'insieme la versione ottocentesca del mosaico della cupola di Aquisgrana può essere considerata conforme all'originale, una serie di dettagli figurativi e cromatici appaiono dubbi e in taluni casi erronei. Già gli esperti chiamati dal governo prussiano avevano mostrato perplessità in proposito, dovendo fare i conti con significative discrepanze nel confrontare la testimonianza di Giovanni Ciampini ${ }^{22}$ con quella di Peter von Beeck, e rilevare alcune omissioni da parte di entrambi in merito all'articolazione iconografica dei singoli soggetti.

L'autore della tavola pubblicata nei Vetera monimenta si è limitato a riprodurre un'esigua porzione della cupola, comprendente soltanto tre degli otto spicchi della volta a padiglione, restituendo al centro la figura del Cristo in trono, in alto una coppia di angeli e in basso sette vegliardi, appena sollevatisi da un trono ritratto accanto a ciascuno di essi. Il busto di Cristo e le due figure alate appaiono immersi in un anello di luce attraversato da alcuni raggi zenitali. In basso, sotto alla Majestas, si scorge un monogramma di Cristo di antica memoria. Tali dati figurativi

19. "Attorno al trono, poi, c'erano ventiquattro seggi e sui seggi stavano seduti ventiquattro vegliardi avvolti in candide vesti con corone d'oro sul capo", Ap. IV, 4.

20. Il motivo che spinse i mosaicisti a raffigurare il Cristo, soggetto principale della grandiosa rappresentazione, non al centro della cupola ma a mezza altezza della calotta, sul lato orientale ("ad Orientem Solem»: P. von Beeck, Aquisgranum, p. 50), è verosimilmente dovuto alla scelta di collocare l'immagine del Salvatore di fronte al trono di Carlo Magno, ubicato nella tribuna occidentale, al fine di stabilire un dialogo visivo fra potere imperiale e autorità celeste: F. Kreusch, Über Pfalzkapelle und Atrium zur Zeit Karls des Grossen, p. 63-66; M. D’Onofrio, Roma e Aquisgrana, p. 128, fig. 71.

2I. Allusione al trono apocalittico (v. supra, nota 19).

22. Le imprecisioni della lettura del Ciampini sono da addurre al fatto che l'autore, non potendosi recare personalmente in loco, si era dovuto affidare alle informazioni fornitegli dal decano del capitolo di Aquisgrana, Johannes Vanderlingen (J.-B. Bethune D’Ydewalle, «Notes sur le projet de restauration de la mosaïque dans la coupole du dôme d'Aix-la-Chapelle», p. 36). 
convergono con la lettura fornita dallo stesso Ciampini, arricchita da indicazioni riguardanti la cromìa dei soggetti :

Tholum etiam, sive ut nostris placet, trullum, in eodem templo conspicuum, columnae sustinent. Depressior illi concameratio contigit musivi operis beneficio, caelum aureum repraesentans, ut in annexa tabula XLI rubris syderum imaginibus interpunctum; media inter haec sydera pro chori conspectu effigies Christum Dominum exhibet, solio insidentem, aureo diademata (cui etiam augustum crucis ornamentum intexitur) coronatum; sinistra Christi manus librum tenet, dextera compositis, more benedicentium, digitis elevatur: at universum corpus talaris tunica, cui paludamentum rubei coloris superimponitur, gemmea fibula apte connexum. Adstant singuli utrinque angeli apertum volumen exhibentes et regis sui numeros alacres stipantes. E tholi centro, luminis in morem erumpunt radii, qui se circum Christi caput diffundunt. Circa thronum locatur sphaera, seu globus, coloribus quinque distinctus, quorum primus throno proximior, est albiculus, secundus venetus, tertius thalassinus, quartus violaceus, quintus janthinus. Haec autem omnia mysterio minime carent, cum desumpta sint ex Apocalypsi cap. 4 ubi: «Sedes posita erat in caelo ...». Imaginibus itaque subtus Christi pedes in zophoro circa trullum locatis nomen appingit hoc ipsum caput apocalypsis, ad cuius normam conformatae videntur. Coronas aureas manibus praeferunt, sedibus relictis, stantes, et candidis vestibus ornatae imitantur $[\ldots]^{23}$.

23. G. Ciampini, Vetera Monimenta, II, p. 134 sq. (per il riferimento al chrismon, v. infra, p. 136). Per facilità di lettura, riportiamo la traduzione in lingua francese di Jean-Baptiste Bethune del brano di Ciampini riportato nel testo: «Les colonnes supportent la lanterne du dôme que rehausse l'éclat de la mosaïque: on y a représenté un ciel d'or semé d'étoiles écarlates: au milieu de ces étoiles, du côté du chœur, on voit l'image de Notre Seigneur Jésus-Christ assis sur un trône; sa tête est entourée d'un nimbe d'or (dans lequel est figuré l'auguste emblème de la croix); de la main gauche le Christ tient un livre, sa main droite est levée dans le geste de la bénédiction: une tunique longue enveloppe tout le corps et une toge de couleur rouge, fixée par une agrafe gemmée, la recouvre. Des deux côtés auprès des épaules, un ange agile montre un livre ouvert. Du milieu de la voûte sortent comme des rayons de lumière qui s'épanouissent autour de la tête du Sauveur. Le trône est placé au centre d'une auréole formée de cinq nuances distinctes, la plus rapprochée du trône est blanchâtre, la seconde azurée, la troisième verdâtre, la quatrième violacée, et la cinquième pourprée. Le sujet représenté se rapporte évidemment au texte du chapitre IV de l'Apocalypse: Et voici, il y avait un trône dans le ciel [...]. Les personnages placés autour de la coupole montrent bien clairement l'intention qu'a eue l'artiste de représenter la vision apocalyptique. En effet, s'étant levés de leurs sièges, 


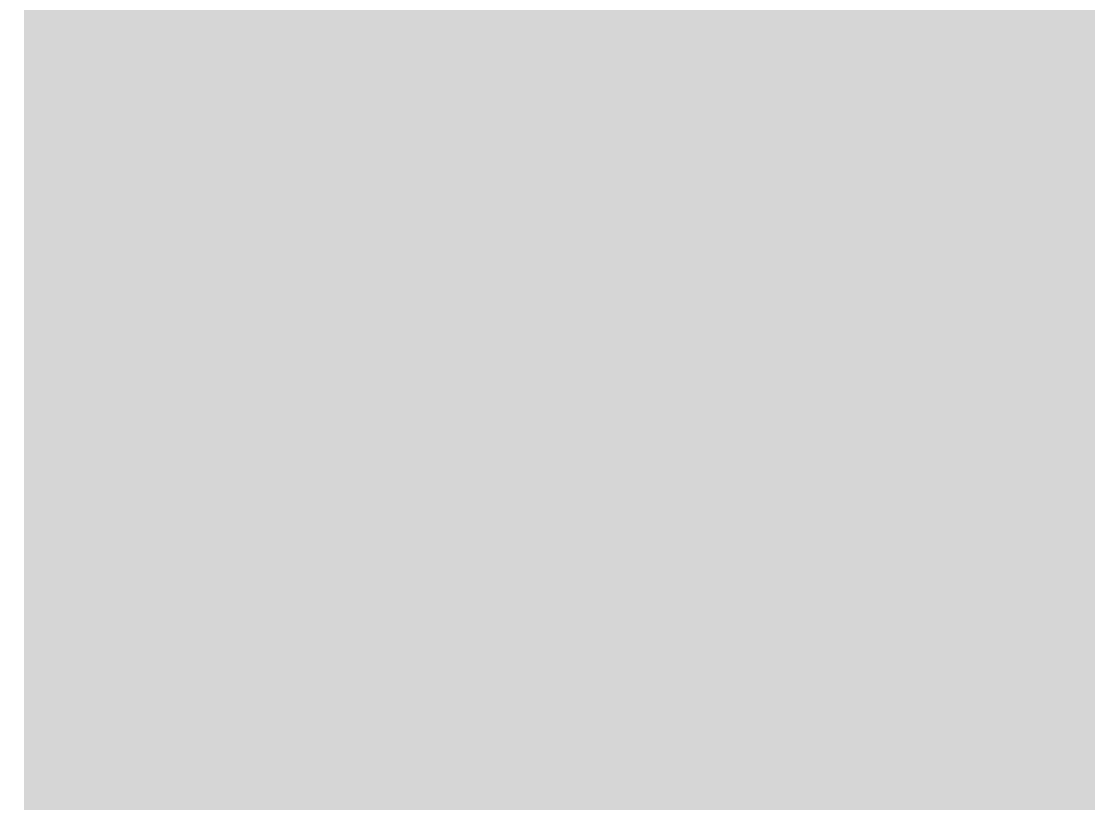

Fig. 3 - Aquisgrana, Cappella Palatina, dettaglio del motivo zenitale della cupola.

Tanto l'immagine dei seggi dei Seniores che quella degli angeli, menzionati da Ciampini e rappresentati nell'incisione pubblicata nel suo libro, non sono state riprodotte nella versione musiva ottocentesca ${ }^{24}$ (fig. 2). Nel primo caso se ne ignora la ragione, visto che il dettaglio trova conferma anche nella lettura di Peter von Beeck ("assurgentes de sedilibus») ${ }^{25}$. Nel secondo caso, come s'è detto, alle figure alate vennero preferiti i quattro simboli degli evangelisti citati da Beeck (fig. 3), soggetto per altro assai più credibile se si pensa all'iconografia della Majestas Domini, di lunga e solida tradizione, ove il Cristo tronante appare di

ils offrent leurs couronnes et sont habillés de vêtements blancs [...]» : J.-B. Bethune D'Ydewalle, "Notes sur le projet de restauration de la mosaïque dans la coupole du dôme d'Aix-la-Chapelle», p. 37 sq.

24. Nella sua proposta di restituzione grafica dell'assetto figurativo della cupola della Cappella Palatina di Aquisgrana, Janina Kalinowska ha rappresentato una schiera di angeli ruotanti attorno alla zona zenitale: J. Kalinowska, "Akwizgran», fig. 14. Cfr. U. Wehling, Die Mosaiken im Aachener Münster und ihre Vorstufen, p. 36 sq., fig. 30.

25. Cfr. il brano di P. von Beek citato infra nel testo. 
solito circondato dal tetramorfo ${ }^{26}$. Altre divergenze fra le descrizioni dei due eruditi seicenteschi riguardano aspetti più marginali, come ad esempio il colore delle stelle, rosse per Ciampini, d'oro per Beeck. Per completezza d'informazione, qui di seguito si riportano anche i passi salienti della lettura del mosaico fornita dall'autore tedesco, che precede la testimonianza dell'italiano di circa settant'anni:

[...] In hoc siquidem opere musivo sedes posita est ad orientem solem, et supra sedem in majestate sedens Salvator ac Servator Christus Dominus pluviali sacro pallio seu talari chlamyde amictus ad eam faciem, quae in Principe Urbium Roma, in divorum Iohannis Lateranensis, Sabae, aliorumque pervetustis ecclesiis visitur. In circuitu sedis quatuor animalia: animal primum simile leoni, et secundum animal simile vitulo, et tertium animal habens faciem quasi hominis, et tertium [sic!] animal simile Aquilae volanti; inde stellae aureae in orbem radiant [...]. Adhuc circulariter contemplari est quatuor et viginti seniores, qui assurgentes de sedibus procidunt ante sedentem in throno, mittentes coronas suas. Et haec de Musaico, quod usque nunc superest ${ }^{27}$.

La stessa commissione di esperti, riunitasi in occasione del ripristino tardo-ottocentesco, aveva dovuto sopperire, inoltre, alla mancanza di notizie relative al soggetto coincidente con il punto zenitale - cui né Beeck né Ciampini danno conto -, optando per il citato medaglione a forma di parasole, probabilmente concepito ispirandosi al modello

26. A.-O. Poilpré, Maiestas Domini, spec. p. 116-123.

27. P. von Beeck, Aquisgranum, p. 50: «Dans cette coupole, du côté de l'Orient, se trouve représenté un trône et sur ce trône est assis dans l'éclat de la majesté divine, le Christ Sauveur revêtu d'un pluvial sacré ou de la longue chlamyde; c'est ainsi qu'il est aussi figuré à Rome, la capitale du monde, dans les basiliques de Saint-Jean de Latran, de Saint-Sabas et dans d'autres très anciennes églises. Autour du trône on voit quatre animaux, le premier ressemble à un lion majestueux, le second à un veau, le troisième à une figure d'homme, et le quatrième représente un aigle aux ailes éployées. Des étoiles d'or brillent tout à l'entour [...]. Il y a en outre, vingt-quatre vieillards qui s'étant levés de leurs sièges, s'avancent vers le Rédempteur assis sur son trône, et lui offrent leurs couronnes. Voilà ce qui existe encore aujourd'hui en mosaïque» (trad. di J.-B. Bethune D’Ydewalle, "Notes sur le projet de restauration de la mosaïque dans la coupole du dôme d'Aix-la-Chapelle», p. 35 sq.). 
di quello della cupola del mausoleo di Santa Costanza, non a caso ben visibile nell'incisione pubblicata, anch'essa, nei Vetera Monimenta ${ }^{28}$.

La questione dell'originario assetto del mosaico della cupola di Aquisgrana torna di attualità negli anni ' 30 del secolo scorso, quando Jean Hubert dà alle stampe inediti documenti d'archivio ${ }^{29}$ - a tutt'oggi non ancora adeguatamente presi in considerazione -, che consentono di mettere in evidenza alcune inesattezze della copia musiva e di formulare nuove riflessioni riguardo al programma primitivo. Nel fondo "Peiresc", conservato presso la biblioteca comunale di Carpentras (Vaucluse), lo studioso francese aveva rinvenuto note manoscritte e disegni databili agli anni 1607-1608, riguardanti l'interno della Cappella Palatina ${ }^{30}$. Si tratta di appunti di viaggio di un testimone oculare, Palamède Fabri, signore di Valavez (1582-1648), recatosi ad Aquisgrana su richiesta del fratello maggiore, Nicolas-Claude Fabri, signore di Peiresc (1580-1637), noto cultore di storia e scienze naturali, animato dall'ardente desiderio di conoscere l'aspetto del monumento carolingio ma impossibilitato a recarvisi perché residente, a quel tempo, nella lontana Provenza ${ }^{31}$.

Dagli appunti di Palamède Fabri si apprende che il cielo trapunto di stelle era in buona parte blu ${ }^{32}$ e non tutto d'oro come il fondo del mosaico moderno, notizia che trova riscontro nella testimonianza di un numero considerevole di tessere di colore azzurro, oltreché a foglia d'oro,

28. G. G. Ciampini, Vetera Monimenta, II, tav. I. L'incisione pubblicata dal Ciampini è una copia di un acquerello di Pietro Santi Bartoli (1635-1700): A. A. Amadio, I mosaici di Santa Costanza, p. 70 sq., fig. 43. Cf. fig. 2, p. 130 dell'articolo di I. Foletti in questo volume.

29. J. Hubert, «Séance du 29 avril».

30. La scoperta del fondo Peiresc, e la prima pubblicazione di altri documenti in esso contenuti, si deve a J. Schopfer, «Documents relatifs à l'art du Moyen Âge contenus dans les manuscrits de N.-C. Fabri de Peiresc à la Bibliothèque de la ville de Carpentras». Cfr. J. Hubert, «Séance du 29 avril», p. 135, n. 3.

31. Così Nicolas-Claude Fabri si rivolge a suo fratello Palamède in una lunga lettera del 1608: "Sur toutes choses que je désirerois de vous, ce seroit que des pays des Estatz [...] vous prinsiez le droict chemin de la ville de Aquisgranum [...]. J'ay un extreme désir de scavoir ce qui est demeuré en estat pour le jourd'huy du tombeau de Charlemagne [...]. Et surtout prenez bien garde s'il n'y a point de mosaïque en l'église dudict Aquisgranum» (cfr. Lettres de Peiresc, VI, p. 682).

32. Palamède Fabri definisce la cupola di Aquisgrana «en mosaïque d'azur estoilée d'or»: J. Hubert, «Séance du 29 avril», p. 136. 
Fig. 4 - Dettaglio di uno dei vegliardi del mosaico della cupola della Cappella Palatina di Aquisgrana, disegno di Palamède Fabri (1607-1608). 
in parte reimpiegate negli stucchi del rivestimento barocco ${ }^{33}$, in parte conservate in stato erratico all'interno di un locale annesso alla Cappella Palatina, almeno fino allo scadere del XIX secolo ${ }^{34}$.

Dal disegno edito da Jean Hubert ${ }^{35}$ (fig. 4), inoltre, si evince chiaramente che nel mosaico carolingio i Vegliardi dell'Apocalisse erano stati raffigurati ciascuno accanto al proprio trono, elemento iconografico documentato, come s'è detto, sia da Ciampini che da Beeck ma non riprodotto nella decorazione musiva ottocentesca. Di grande interesse è anche un altro schizzo del medesimo taccuino di viaggio, pubblicato più tardi da Erich Stephany ${ }^{36}$ e poi, in anni più recenti, da Jerzy Miziolek e Ulrike Wehling ${ }^{37}$, riproducente la zona sommitale della volta (fig. 5). Con tratto rapido ma efficace il signore di Valavez ha disegnato in primo piano la sagoma di uno dei seniores, levante il braccio destro in direzione del cielo stellato. Quest'ultimo è stato rappresentato appena al disopra della mano del vegliardo, in scala più piccola, quasi a voler suggerire una visione prospettica della cupola dal basso verso l'alto. Il firmamento di stelle, al centro della calotta, appare ripartito in tre zone concentriche. Quella inferiore, abitata dall'immagine del Cristo, risulta omogenea. I due anelli superiori, di altezza più esigua, sono invece attraversati da una serie di fasci radianti riscontrabili, come s'è detto, anche nel disegno

33. «La mosaïque du dôme a cessé d'exister au siècle dernier. Déjà notablement altérée par l'incendie violent de 1656, elle a été complètement détruite par la main barbare d'un artiste étranger qui, de 1720 à 1730, a préféré la remplacer par des stucs du goût le plus équivoque et n'en a conservé le souvenir que par des panneaux en cubes d'émail bleu ou doré, fixés de manière à rehausser ses décors blancs": X. Barbier de Montault, «La mosaïque du Dôme à Aix-la-Chapelle», p. 289.

34. "Dans la salle du chapitre trois vastes corbeilles sont également remplies de débris. J'ai passé beaucoup de temps à examiner minutieusement leur contenu et je n'y ai guère trouvé que des émaux bleus ou dorés": Ibidem, p. 318. Si conferma esatta, quindi, l'intuizione di Xavier Barbier de Montault, che credeva nell'esistenza di un'ampia fascia di cielo blu, sormontata da un anello sommitale a fondo oro: "Je ne me croirai pas loin de la vérité en affirmant que la zone d'or a dû être élevée au-dessus d'une zone bleue. D'ailleurs cette zone bleue est pour ainsi dire nécessitée et par les monuments analogues et par la convenance hiérarchique...»: Ibidem, p. 293. Cfr. anche U. Wehling, Die Mosaiken im Aachener Münster und ihre Vorstufen, p. 19-23.

35. J. Hubert, «Séance du 29 avril», fig. 2.

36. E. Stephany, «Die Bilder aus Aachen für Monsieur Peiresc, 1607-1608 », p. 68, fig. 3.

37. J. Miziolek, "When our sun is risen", p. 16, fig. 16; U. Wehling, Die Mosaiken im Aachener Münster und ihre Vorstufen, p. 15, fig. 6. 
Fig. 5 - Dettaglio del motivo alla sommità della cupola della Cappella Palatina di Aquisgrana, disegno di Palamède Fabri (1607-1608). 
del Ciampini (fig. 1) : il particolare sembrerebbe confermare la presenza di raggi luminosi provenienti da un medaglione zenitale, cui pare far riferimento, in effetti, il semicerchio sommitale raffigurato nella tavola dei Vetera monimenta. Al centro del clipeo, Palamède Fabri ha tracciato un breve tratto rettilineo che cade a piombo verso terra. Il dettaglio si riferisce, assai probabilmente, al lampadario in bronzo dorato donato da Federico Barbarossa al capitolo di Aquisgrana nel 1165 e tuttora conservato, nella medesima posizione, all'interno della Cappella Palatina. Per quanto riguarda il contenuto figurativo del medaglione sommitale, il disegno di Peiresc non ci è d'aiuto, dal momento che al suo interno non è raffigurato nulla. Nel mosaico ottocentesco, s'è detto, il centro della cupola è stato decorato con il motivo del parasole, soluzione possibile ma del tutto arbitraria, priva com'è di elementi probanti.

Sul tema zenitale, in passato, alcuni studiosi hanno proposto ipotesi alternative a nostro parere non molto persuasive. Hermann Schnitzler aveva ipotizzato che all'interno del clipeo sommitale vi fosse originariamente un Agnus Dei ${ }^{38}$. La tesi dello studioso si basava sull'idea che l'immagine del Cristo benedicente fosse un'aggiunta posteriore, dovuta al citato intervento d'installazione del lampadario ad opera dell'imperatore Federico, oppure ad un incendio documentato nel $1224^{39}$. La teoria di Schnitzler appare, però, a ben riflettere, assai poco credibile, sia per la difficoltà di immaginare un intervento musivo tanto ardito nei secoli centrali del Medioevo, sia perché i vegliardi del registro inferiore sono tutti orientati verso est e non protesi verso il medaglione centrale.

Wolfgang Schöne, dal canto suo, aveva suggerito l'ipotesi che il clipeo centrale accogliesse la colomba dello Spirito Santo ${ }^{40}$, mentre Bianca Kühnel aveva pensato alla presenza della dextera Domini ${ }^{41}$. Anche queste due proposte, tuttavia, appaiono poco convincenti: nessun riferimento al simbolo trinitario né alla mano divina emerge dalla lettura fornita da Ciampini e Beeck, né dagli appunti del fondo Peiresc. Oltre tutto risulta piuttosto inverosimile che l'ancoraggio del lampadario

38. H. Schnitzler, «Das Kuppelmosaik der Aachener Pfalzkapelle».

39. Ibidem, p. 38. Cfr. U. Wehling, Die Mosaiken im Aachener Münster und ihre Vorstufen, p. 34-36; J. Miziolek, "When our sun is risen», p. 9.

40. W. Schöne, «Die künstlerische und liturgische Gestalt der Pfalzkapelle Karls des Großen in Aachen", p. 102; Cfr. J. Miziolek, "When our sun is risen", p. 9.

4I. B. Kühnel, From the earthly to the heavenly Jerusalem, p. 131. 
commissionato dal Barbarossa sia stato eseguito in corrispondenza di un soggetto iconico di così grande valenza simbolica.

E' pertanto più ragionevole pensare che al culmine del mosaico carolingio vi sia stato, fin dall'origine, un motivo aniconico alludente alla luce divina, degradante verso l'azzurro del cielo inferiore attraverso anelli concentrici e fasci radianti di tessere d'oro.

Simone Piazza

Université Paul-Valéry Montpellier, CEMM - EA 4583 


\section{BIBLIOGRAFIA}

Alibert, Dominique, «La majesté sacrée du roi: images du souverain carolingien", Histoire de l'art, 5-6 (1989), p. 23-36.

Amadio, Adele Anna, I mosaici di Santa Costanza. Disegni, incisioni e documenti dal XV al XIX secolo, Roma, De Luca, 1986.

Barbier de Montault, Xavier, "La mosaïque du Dôme à Aix-laChapelle», Annales archéologiques, 26 (1869), p. 285-338.

Beеcк, Peter von, Aquisgranum. Sive Historia Aquisgrani, Aachen, 1620 (rist. anast. "Kessinger Publishing», 2010).

Belting, Hans, «Das Aachener Münster im 19. Jahrhundert. Zur ersten Krise des Denkmal-Konzepts ", Wallraf-Richartz-Jahrbuch, 45 (I984), p. 257-289.

Bethune D’Ydewalle, Jean-Baptiste, "Notes sur le projet de restauration de la mosaïque dans la coupole du dôme d'Aix-la-Chapelle», Messager des sciences historiques ou Archives des arts et de la bibliographie de Belgique, (1876), p. 31-51.

Binding, Günther, "Die Aachener Pfalz Karls des Grossen als archäologisch-baugeschichtliches Problem», Zeitschrift für Archäologie des Mittelalters, 25-26 (1997-1998), p. 63-85.

Bordi, Giulia, "Il busto di Cristo, i quattro simboli degli evangelisti, i ventiquattro Seniores e gli apostoli Pietro e Paolo", in L'orizzonte tardoantico e le nuove immagini (312-468), a cura di Maria Andaloro, Milano, Jaca Book, 2006, p. 395-402.

—, «L'Agnus Dei, i quattro simboli degli evangelisti e i ventiquattro Seniores nel mosaico della facciata di San Pietro in Vaticano", in L'orizzonte tardoantico e le nuove immagini (312-468), a cura di Maria Andaloro, Milano, Jaca Book, 2006, p. 416-418.

Ciampini, Giovanni Giustino, Vetera Monimenta, Roma, Typographia Bernabò, 1699.

Clemen, Paul, Die romanische Monumentalmalerei in den Rheinlanden, Düsseldorf, Schwann, 1916.

D’Onofrio, Mario, Roma e Aquisgrana, Roma, Ed. Rari Nantes, 1983. 
Dutton, Paul Edward, Charlemagne's mustache and other cultural clusters of a dark age, New York, Palgrave Macmillan, 2004.

Euw, Anton von, "Karl der Grosse als Schüler Alkuins, das Kuppelmosaik des Aachener Domes und das Maiestasbild in Codex C 80 der Zentralbibliothek Zürich ", Zeitschrift für schweizerische Archäologie und Kunstgeschichte, 61 (2004), p. 1-20.

Falkenstein, Ludwig, "Charlemagne et Aix-la-Chapelle», Byzantion, 61.1 (1991), p. 231-289.

Gaehde, Joachim E., "La decorazione. Le miniature», in Commentario storico, paleografico, artistico, critico della Bibbia di San Paolo Fuori le Mura, Roma, Istituto Poligrafico e Zecca dello Stato, 1993, p. 235-328.

Grimme, Ernst Günther, Dom zu Aachen. Architektur und Ausstattung, Aachen, Einhard-Verl., 1994.

Heckes, Pia, "Die Mosaiken Hermann Schapers im Aachener Münster", Aachener Kunstblätter, 52 (1984), p. 187-230.

Hubert, Jean, "Séance du 29 avril», Bulletin de la Société Nationale des Antiquaires de France, 1936, p. 132-140 (rist. con il titolo «La mosaïque disparue de la chapelle palatine de Charlemagne à Aix", in Nouveau recueil d'études d'archéologie et d'histoire, de la fin du Monde antique au Moyen Âge, Genève, Droz, 1985, p. 301-309).

Kalınowska, Janina, "Akwizgran: Przyczynek do poznania pierwotnego programu ikonograficznego dekoracji mozaikowej Kaplicy palacowej karola wielkiego ", Folia Historiae Artiura, 18 (1982), p. 5-23.

Kreusch, Felix, Über Pfalzkapelle und Atrium zur Zeit Karls des Grossen, Aachen, Wilhelm Metz, 1958 (Dom zu Aachen. Beiträge zur Baugeschichte 4).

KüHnel, Bianca, From the earthly to the heavenly Jerusalem, Roma, Herder, 1987.

Lettres de Peiresc, VI, éd. par Philippe Tamizey de Larroque, Paris, L. Techener, 1896.

Miziolek, Jerzy, «When our sun is risen : observations on eschatological visions in the art of the first millenium: II", Arte cristiana, 83.766 (1995), p. 3-22.

Nordhagen, Per Jonas, "Mosaico", in Enciclopedia dell'arte medievale, VIII, Roma, Treccani, 1997, p. 563-574. 
Osborne, John, "The Jerusalem temple treasure and the church of Santi Cosma e Damiano", Rome Papers of the British School at Rome, 76 (2008), p. 173-181.

Piazza, Simone, Allo zenit della cupola. L'eredità dell'oculus nell'arte cristiana fra Medio Evo latino e Bisanzio, Roma, Campisano Editore, 2018.

PoIlpré, Anne-Orange, Maiestas Domini: une image de l'Église en Occident ( $V^{e}-I X^{e}$ siècle), Paris, Éd. du Cerf, 2005.

Schnitzler, Hermann, "Das Kuppelmosaik der Aachener Pfalzkapelle», Aachener Kunstblätter, 29 (1964), p. 17-44.

Schöne, Wolfgang, "Die künstlerische und liturgische Gestalt der Pfalzkapelle Karls des Großen in Aachen ", Zeitschrift für Kunstwissenschaft, 15 (1961), p. 97-148.

Schopfer, Jean, "Documents relatifs à l'art du Moyen Âge contenus dans les manuscrits de N.-C. Fabri de Peiresc à la Bibliothèque de la ville de Carpentras", Bulletin archéologique du Comité des travaux historiques, (1899), p. 330-395, tavv. XV-XIX.

Schrade, Herbert, "Zum Kuppelmosaik der Pfalzkapelle und zum Theoderich-Denkmal in Aachen", Aachener Kunstblätter, 30 (1965), p. 49-58.

Stephany, Erich, "Die Bilder aus Aachen für Monsieur Peiresc, 16071608 ", Zeitschrift des Aachener Geschichtsvereins, 69 (1957), p. 67-70.

THunø, Erik, " "Living stones” of Jerusalem: the triumphal arch mosaic of Santa Prassede in Rome", in Visual constructs of Jerusalem, dir. by B. Kühnel, G. Noga-Banai, H. Vorholt, Turnhout, Brepols, 2014, p. 223-230.

Vanderputten, Steven, "La Chapelle Palatine à Aix-la-Chapelle et l'héritage classique", Bulletin de l'Institut Historique Belge de Rome, 70 (2000), p. 5-38.

Wehling, Ulrike, Die Mosaiken im Aachener Münster und ihre Vorstufen, Koln, Rheinland-Verl., Köln, 1995 (tesi di dottorato, Università di Bonn, 1992).

Zаnотto, Rita, "Mosaici, e sectilia, reimpiegati da Ravenna ad Aquisgrana: contesto storico e questioni aperte", in Atti del VII Colloquio dell'Associazione italiana per lo studio e la conservazione 
del mosaico, a cura di A. Paribeni, Ravenna, Edizioni del Girasole, 2001, p. 587-594.

Crediti fotografici:

Fig. 1: Ciampini, Giovanni Giustino, Vetera Monimenta, Roma, Typographia Bernabò, 1699, II, tav. XLI.

Fig. 2-3: Cliché de l'auteur.

Fig. 4: Hubert, Jean, "Séance du 29 avril», Bulletin de la Société Nationale des Antiquaires de France, 1936, fig. 2.

Fig. 5: Stephany, Erich, "Die Bilder aus Aachen für Monsieur Peiresc, 1607-1608», Zeitschrift des Aachener Geschichtsvereins, 69 (1957), fig. 3 . 